\title{
$\varangle \quad$ Las fuentes de información del patrimonio histórico
}

\section{Isabel Ortega Vaquero}

Centro de Documentación del IAPH

\section{CARACTERÍSTICAS DE LA DOCUMENTACIÓN DEL PATRIMONIO HISTÓRICO}

La Información y la Documentación son de vital importancia para el Conocimiento del Patrimonio Histórico, para el desarrollo de una tutela integral. Afirmación que podemos encontrar en las Disposiciones Legales sobre Patrimonio Histórico:

La Ley 16/1985 de Patrimonio Histórico Español recoge la importancia que el conocimiento del Patrimonio Histórico tiene para su protección y conservación, por parte de la Administración y para la sociedad en general, y la responsabilidad de la Administración para instrumentar medidas que permitan el acceso a la información como factor fundamental para una política eficaz de los Bienes Culturales.

Esta idea está recogida en el Plan General de Bienes Culturales estableciendo que "debemos perfeccionar nuestro conocimiento acerca de los Bienes Culturales en Andalucía y mejorar la base científica de nuestra información sobre Patrimonio Histórico", destacando la necesidad de cumplir una función informativa tanto para la Administración como para el investigador o el público en general, debido a la falta de accesibilidad de la información producida.

En este mismo sentido, la Ley |/|99| de Patrimonio Histórico de Andalucía confiere al Catálogo General, como instrumento básico de salvaguarda, tres funciones fundamentales, una función de Conocimiento, una función Instrumental y una función Informativa, facilitando el conocimiento de los Bienes a través de un sistema de fácil consulta y accesibilidad, que usando las nuevas técnicas informáticas permitan una más exacta valoración de los diversos Bienes y la interacción entre dichos Bienes.

Por último, el Decreto 107/89, de 16 de mayo (BOJA de 13/6/89) de creación del Instituto Andaluz del Patrimonio Histórico establece como uno de sus objetivos fundamentales "el conocimiento del Patrimonio Histórico mediante su documentación", porque difícilmente podrán protegerse y conservarse los Bienes Culturales sin un conocimiento exhaustivo de ellos.
Pero el conocimiento del Patrimonio Histórico a través de la documentación e información está condicionado por una serie de características o dificultades que son en líneas generales:

I. La amplitud del Patrimonio Histórico, en cuanto a su cuantificación y tipologías, y la diversidad del ámbito temático, ya que abarca diferentes temáticas o disciplinas, desde la arquitectura a la museología, de la ordenación urbana a la conservación y restauración, desde la arqueología a la historia, etc., pero sin una unidad doctrinal que las aglutine.

2. La documentación sobre Patrimonio Histórico responde a una variedad y diversidad de tipologías documentales, desde las monografías a las publicaciones periódicas, desde las actas de congresos a las tesis, desde la documentación gráfica a la cartografía, desde la documentación inédita de archivos a las bases de datos, etc.

3. En la producción de documentación e información intervienen diferentes agentes, que de forma retrospectiva o actual, a nivel internacional, nacional, autonómico o local, intervienen en la gestión, investigación o difusión del Patrimonio Histórico.

4. La localización de la documentación se encuentra ubicada en diferentes instituciones, de variada naturaleza (bibliotecas, archivos, fototecas, etc), de diferente dependencia institucional (administración estatal, autonómica, entidades privadas, etc.), de diferentes ámbitos geográficos, etc.

5. La falta de una sistematización de la documentación, y la deficiente difusión de la información.

Por ello, la Información utilizada para la gestión del Patrimonio Histórico ha sido fragmentaria, inconexa, repetitiva, desvertebrada y soportada en métodos tradicionales del tratamiento de la misma, presentando una serie de carencias.

El punto de partida de estas Carencias es una concepción restringida del conocimiento necesario para la tutela del Patrimonio Histórico, ya que se ha entendido como el paso previo a la protección, y no como un conocimiento integral paralelo a una tutela integral, desde la protección a la difusión, hecho que afecta a dos aspectos: 
a. Deficiencias en la gestión de los bienes culturales, que vienen propiciadas por la inadecuada organización interna de cada una de las instituciones, así como la desconexión de las tareas, que conllevan una duplicidad de trabajos, un descontrol de la documentación producida, y la permanencia de unos esfuerzos individualizados, a veces dispares o repetitivos, ocasionada por la inexistencia de una coordinación entre los diferentes organismos relacionados con la problemática de los bienes culturales: educación, turismo, investigación y desarrollo tecnológico, ordenación de territorio y urbanismo, medio ambiente, etc.

b. Deficiencias en la documentación e información sobre el Patrimonio Histórico, producidas por el desconocimiento real de la información sobre Patrimonio Histórico; la falta de trabajos sistemáticos de organización y tratamiento de la documentación, que han provocado el general desconocimiento de los bienes culturales; la inaccesibilidad de la información, ante la inexistencia de programas o proyectos para la elaboración de productos y servicios de información, que facilitara la recuperación y difusión de la información sobre Patrimonio Histórico; la pervivencia de unos procedimientos manuales en el tratamiento y difusión de la información; la desconexión informativa entre diferentes organismos relacionados con los bienes culturales; el escaso desarrollo de la documentación gráfica del Patrimonio Histórico: fotografías, cartografía, dibujos, planimetrías,...; y la escasa aplicación de las nuevas tecnologías al conocimiento del Patrimonio Histórico.

Todo ello imposibilita ofrecer una información unitaria y completa, a la vez que impide avanzar en nuestro conocimiento sobre los Bienes Culturales, ya que un paso es conocer toda la documentación generada por los diversos agentes que de forma retrospectiva o actual se han dedicado a gestionar o investigar alguno de los aspectos incluidos en la temática del Patrimonio Histórico.

Por ello, se considera prioritario la cualificación del proceso de captación de la información mediante nuevos procedimientos, la cualificación en el tratamiento documental e informático y la implantación de unos servicios de información eficientes, porque el Patrimonio Histórico ha sido un gran desconocido durante mucho tiempo debido a su dimensión excepcional y a la inexistencia de una labor de desarrollo sistemático de los instrumentos y técnicas de documentación.

La resolución de las carencias detectadas en la documentación e información del Patrimonio Histórico reportaría una serie de Beneficios, en cuanto a la correcta planificación y gestión de la política cultural, contando con la información como soporte fundamental en la toma de decisiones y evitando la duplicación de esfuerzos por el desconocimiento; la racionalización de los procesos de producción y organización de la información; la disponiblidad rápida, puntual y actualizada de toda la información existente sobre un Bien determinado, y su relación con otros bienes; y la accesibilidad a las fuentes de información producidas, de cualquier tipología y en cualquier soporte.

La documentación y la información aplicadas al Patrimonio Histórico debemos entenderlas como una medida de prevención ante los riesgos de los bienes culturales, como un instrumento para la toma de decisiones en el Patrimonio Histórico, como un medio al servicio de los técnicos, científicos, investigadores y profesionales que actúan y estudian en el Patrimonio Histórico, y como un instrumento para devolverle a la sociedad el legado histórico. Por ello, la información del Patrimonio Histórico debe tener un carácter de integración y coordinación, huyendo de la visión fragmentaria, y considerando al Patrimonio Histórico como un sistema de relaciones. Pero, existe una gran dificultad para definir los límites y el campo del Patrimonio Histórico, por ello es necesario encontrar un método integrado de las distintas disciplinas que faciliten su comprensión, porque el campo del patrimonio no es de ninguna de ellas, sino que deben aunarse para su comprensión global. Hay que ampliar el concepto disciplinar, y entender que en el Patrimonio Histórico se encuentran implicadas múltiples y diversas ramas del saber, comprender que la tutela del patrimonio, entendida como el proceso integral para su conocimiento, exige una labor multidisciplinar e interdisciplinar.

Por otro lado, cualquier proyecto de documentación ha de estar enmarcado dentro de una metodología y una normativa fijada desde los centros de documentación y desde los planes generales de investigación y documentación, de ámbito regional, nacional o internacional. Además es necesario un trabajo interdisciplinar en el campo del patrimonio, porque cada vez es más difícil la separación entre disciplinas, y el trabajo del historiador, el arqueólogo, el etnólogo, el científico o el documentalista se complementan con el restaurador o el arquitecto y con el gestor o el conservador.

\section{LAS FUENTES DE IINFORMACIÓN DEL PATRIMONIO HISTÓRICO}

Una vez delimitado el ámbito temático y expuestas las características que presenta la documentación e información relativa a Patrimonio Histórico, se plantea como una necesidad la localización y recopilación de las fuentes de información, ya que una adecuada protección y conservación del Patrimonio Histórico sólo puede ser planificada y realizada desde el conocimiento de la realidad sobre la que se actúa. Por esta razón la documentación e información es un factor importantísimo que ha de estar presente en el inicio, en todo el proceso, y en su término, porque no se puede proteger ni conservar sin investigar.

Las fuentes de información según Gloria Carrizo son los medios y procedimientos propios de la ciencia de la documentación para localizar y distribuir la información, y las define como "los materiales o productos, originales o elaborados, que aportan noticias o testimonios a través de los cuales se accede al conocimiento, cualquiera que éste sea; y que pueden ser 
monumentos, documentos, libros o productos de ordenador, todo aquello que suministre una noticia, una información o un dato". Esta consideración pone de manifiesto la amplitud del concepto de fuentes de información.

Las fuentes de información se sitúan al principio y al final de la cadena documental. Al principio con la búsqueda y localización de la información, y con la selección y adquisición o captación de la información; y al final a través de la difusión de la información.

\section{Búsqueda y Localización}

Consistente en conocer y localizar los documentos que se producen en los ámbitos temáticos y geográficos prefijados según nuestro ámbito de interés. Si el concepto de Patrimonio Histórico se refiere a los bienes jurídicamente protegidos, la labor de las fuentes de información debe ir dirigida en primer lugar a recopilar la documentación e información que se ha generado sobre dichos bienes, desde los expedientes de declaración y proyectos de restauración, a la bibliografía específica, el material gráfico producido sobre ellos, etc., y en segundo lugar nuestro ámbito debe ser más amplio recopilando aquélla información relacionada con las áreas de conocimiento, centrales y relacionadas, es decir la documentación sobre la arquitectura, la arqueología, los museos, la pintura, etc., generalmente recogida en las fuentes bibliográficas, así como en otros documentos como informes, tesis, normas, etc.

\section{Objetivos}

El objetivo genérico que se plantea es la localización, recopilación y sistematización de las fuentes de información que se han producido sobre Patrimonio Histórico. Es decir, buscar los documentos que contengan información sobre Patrimonio Histórico, para ello, en primer lugar necesitamos conocer los documentos secundarios que nos aportan la información de forma abreviada y que nos sirven de puente para llegar en definitiva a los documentos originales, cualquiera que sea su soporte, desde los documentos textuales a los iconográficos (mapas, planos, fotografías,...), audiovisuales, o informáticos.

Este objetivo genérico debe responder a una doble vertiente

I. Proporcionar la localización y captación de la documentación e información producida en la gestión del Patrimonio Histórico, es decir, aquélla documentación que se ha producido como consecuencia de cualquier acción de la tutela, desde la protección con la elaboración de inventarios y catálogos de bienes, a la conservación con los proyectos de restauración, a la investigación o a la gestión. Estas fuentes se refieren tanto, y de forma fundamental, a la documentación de archivos (textual, fotográfica, planimétrica, etc.) como a las fuentes bibliográficas relativas a difundir actuaciones o estudios sobre los Bienes Culturales.

2. Facilitar otras informaciones, generalmente bibliográficas, que cualifique las investigaciones, estudios, planifi- cación de actuaciones, etc., relativa a teorías, métodos, nuevos avances técnicos, normas, noticias de prensa, disposiciones legales, etc., de las diferentes disciplinas que están implicadas en el conocimiento del Patrimonio Histórico.

\section{Finalidad}

La finalidad última es cumplir una función informativa, consistente en la disponibilidad de las fuentes de información sobre Patrimonio Histórico, y no en la posesión de los fondos documentales pertenecientes a otras instituciones, a través de la localización y captación sistemática y/o puntual de la información contenida en las fuentes existentes (bibliográficas, textuales, gráficas, etc.), con el fin de posibilitar la disponibilidad y acceso a las fuentes de información del Patrimonio Histórico en general y de los Bienes Culturales en particular, al servicio de las necesidades de la administración, los investigadores, entidades y público en general.

Para determinar este aspecto de forma precisa sería necesario proceder a un Estudio de Usuarios, estableciendo los diferentes grupos, con sus características, especificidades y sobre todo con las expectativas y necesidades de información. Pero, de forma genérica, podemos fijar tres grandes grupos, que a la vez sirven a tres finalidades:

I. La Administración, en todas sus vertientes, local, autonómica y estatal; para la mejora de la toma de decisiones, la gestión y la tutela del patrimonio.

2. Los investigadores y estudiosos del patrimonio; para la cualificación de las investigaciones sobre patrimonio.

3. El público en general; para el acercamiento del patrimonio a la sociedad.

\section{Metodología}

Para la consecución de los objetivos reseñados se plantean diversos criterios de planificación:

- Atendiendo a la tipología de las fuentes de información (bibliográficas, documentos de archivos, documentación gráfica,...).

- Según un criterio o factor institucional (archivos históricos y administrativos, bibliotecas, fototecas, hemerotecas,...).

- De acuerdo a los ámbitos temáticos (arquitectura, arqueología, etnología, escultura,...).

- Atendiendo a la tipología de los bienes culturales (patrimonio arqueológico, patrimonio documental, patrimonio arquitectónico,...) etc.

Las diferentes alternativas no se presentan de forma excluyente, sino que pueden abordarse mediante una priorización y faseado temporal que las aglutine en el desarrollo global de la localización de las fuentes de información del Patrimonio Histórico. 
La metodología general a seguir, en grandes líneas, es:

I. Conocer aquéllas instituciones, organismos, entidades, etc., que contengan o elaboren información de interés sobre Patrimonio Histórico, atendiendo a dos aspectos:

- Instituciones que contengan fondos de interés: bibliotecas, archivos, fototecas, centros de documentación, etc.

- Organismos o entidades que realicen alguna actividad de interés para el patrimonio: asociaciones, fundaciones, entidades privadas, etc.

2. Definir la información necesaria para el conocimiento del Patrimonio Histórico, reseñando algunas características generales y específicas de cada tipo documental, de acuerdo con la clasificación inicial y básica de las fuentes de información, en dos grandes grupos:

- Fuentes Bibliográficas, que son libros, artículos y una larga serie de productos elaborados que permiten obtener información.

- Fuentes Documentales, que tienen una asimilación a la investigación histórica, a la búsqueda y conocimiento de las fuentes de la historia.

3. Localizar y conocer los productos de información producidos sobre Patrimonio Histórico, tanto si se trata de productos especializados en algunas de las temáticas abarcadas, como si son productos multidisciplinares con alguna información en nuestro ámbito, centrándonos en los instrumentos de búsqueda y localización de la información existente sobre Patrimonio Histórico.

La primera labor que se realiza en el campo documental es la búsqueda de la documentación existente, es una labor previa a cualquier investigación o actuación. La documentación sobre patrimonio es de muy variada naturaleza y aporta información muy diversa, como por ejemplo:

- Bibliografía: los manuales, las monografías y los artículos de revistas especializadas aportan una visión previa, pero no existe en España una bibliografía sobre Patrimonio Histórico actualizada, sino que sólo existen bibliografías parciales.

- Documentación escrita inédita: en los textos conservados en archivos encontramos valiosas informaciones sobre los bienes culturales, como las restauraciones, destrucciones parciales, cambios de localización, etc. Pero la documentación sobre patrimonio se encuentra muy dispersa, repartida en numerosos archivos, a veces sin inventariar ni catalogar.

- Fotografías: la documentación fotográfica tiene una importancia trascendental para el conocimiento de los bienes culturales, permitiendo evaluar los cambios sufridos por el bien en cuanto a deterioros, restauraciones, alteración del entorno, cambios de ubicación, etc. Pero también los documentos fotográficos se encuentran muy dispersos en distintos archivos.

- Planos y proyectos de restauración: es fundamental conocer las actuaciones realizadas sobre los bienes culturales a través de los proyectos de intervención, que además contienen levantamientos planimétricos en el caso de monumentos. Esta documentación también se encuentra dispersa y a veces ilocalizable, no existiendo, además, una publicación o base de datos que recoja toda esta documentación.

- Inventarios: la realización de inventarios del patrimonio ha sido una de las constantes preocupaciones de la administración desde principios de siglo, pero nunca ha llegado a materializarse en unos inventarios operativos.

- Bases de datos: las bases de datos sobre Patrimonio Histórico en nuestro país son escasas. El único conjunto sistemático es el realizado por el Ministerio de Cultura a través de la red PIC, aunque se encuentra la información sin actualizar. Además numerosas comunidades autónomas están elaborando sus propios inventarios, como el caso de Valencia, o de Andalucía.

- Recursos de información en redes informáticas: principalmente en Internet, donde podemos encontrar información diversa de organizaciones internacionales y nacionales relacionadas con el Patrimonio Histórico, como UNESCO, ICOM, ICCROM, Departamentos Universitarios, Bibliotecas de arte y arquitectura, Red $\mathrm{ARCH}$ NET que reúne los recursos de arqueología, etc.

Para la localización de la documentación e información deberemos basarnos en las obras y productos de información ya elaborados, que nos sirvan de instrumentos básicos de información y de instrumentos de búsqueda y localización de la información, tales como enciclopedias, diccionarios, inventarios, catálogos, directorios, guías, bibliografías, bases de datos, etc.

Por otro lado, es necesario recopilar la documentación e información generadas en cualquier actividad sobre los bienes culturales: intervenciones, excavaciones, etc. Todo proceso viene marcado por la necesidad de dejar constancia de lo realizado, así como de aquellas características del bien cultural que salen a la luz en la actuación; por ello es fundamental planificar el proceso de documentación según un método normalizado y sistemático, recogiendo los datos del proceso, y almacenando y conservando esa documentación. Es decir, esta etapa integra dos partes: unos estudios previos, tanto de carácter histórico-documental como científico-técnico, y la propia documentación del proceso.

\section{Selección y Adquisición}

Consiste, una vez localizadas, en la selección de aquellas fuentes de información que cubren nuestro ámbito específico y las necesidades y demandas de nuestros usuarios, así como su posterior adquisición a través de diferentes medios (compra, intercambio, donación, etc.), o bien la captación de la información para la realización de productos informativos como bibliografías, impresas o informatizadas. 
La documentación generada en todo el proceso debe recogerse por el centro de documentación, que debe contemplar la información de origen diverso y sobre soportes diferentes, siendo la base la información de los bienes culturales que debe remitir a cuanta información exista, fuentes de información bibliográficas, documentales, fotográficas, cartográficas, etc., que no debe limitarse a los fondos existentes físicamente en el centro sino a toda la documentación localizable.

Asimismo toda la información debe ser informatizada, a través de la creación de las bases de datos correspondientes. Por otro lado, el Centro de Documentación debe ser capaz de generar aquella documentación que no exista, a través de la planificación y realización de proyectos de investigación y desarrollo basados en las necesidades del Patrimonio, con el fin de ampliar el conocimiento de los Bienes Culturales.

\section{Difusión de la Información}

Permite la distribución de los documentos proporcionando la información específica que cada usuario necesite, a través de diferentes medios. El Centro de Documentación debe difundir la información que posee, a través de servicios y productos de información dirigidos a los usuarios específicos y a la sociedad en general.
Es importante destacar el papel de las fuentes secundarias (bibliografías, los boletines de índices, de resúmenes y de sumarios, los catálogos de las bibliotecas, de los editores y libreros, los catálogos colectivos, las bases de datos referenciales, tanto en línea como en discos ópticos, etc.) en las fases reseñadas de la cadena documental, ya que cumplen dos funciones fundamentales, una como repertorios que facilitan la búsqueda, localización y selección de los documentos, y otra como instrumentos para la recuperación retrospectiva de la información y la Difusión Selectiva de la Información.

Además de la labor interna del centro de documentación, es fundamental el desarrollo de programas de cooperación con otras instituciones, así como la inclusión de su programación dentro de planes de información de ámbito regional, nacional e internacional. En este sentido, señalar que la Ley 16/85 en su artículo 35 establece que para la protección de los bienes integrantes del Patrimonio Histórico Español y al objeto de facilitar a los ciudadanos el acceso a los mismos, fomentar la comunicación entre los diferentes servicios y promover la información necesaria para el desarrollo de la investigación cientííca y técnica, se formularán periódicamente Planes Nacionales de Información sobre el Patrimonio Histórico Español.

\section{Bibliografía}

AGUILAR CORREDERA, Francisco, PAVÓN RENDÓN, Joaquín, VALVERDE CUEVAS, Fernando. Régimen jurídico del Patrimonio Histórico en Andalucía. Sevilla: Consejería de Cultura. Junta de Andalucía, D.L. 1995 (Textos Legales)

ÁLVAREZ ÁLVAREZ, J.L. Estudios sobre el Patrimonio Histórico Español y la Ley de 25 de Junio de 1985. Madrid: Cívitas, 1989

AMAT NOGUERA, Nuria. Documentación científica y nuevas tecnologías de la información. Madrid: pirámide, 1988

AMAT NOGUERA, Nuria. Técnicas documentales y fuentes de información. Barcelona: Bibliograf, 1979

ANSELMI, Laura, GHELARDI, Mariagrazia. Cooperación europea en el campo de la arquitectura y disciplinas relacionadas. En: Preconferencia de bibliotecas de arte. IFLA Section of Art Libraries. Barcelona, |8-21 de agosto de 1993, págs. 103-107

ARREGI Y AZPEITIA, Gurutzi de. Metodología para el estudio de la etnografía religiosa: ermitas y cofradías. En: OHITURA. Cuadernos de Etnografia alavesa, nº 5, 1987, pp. 123134

ATHERTON, P. La comunicación de la información: manual para senvicios y sistemas de información. París: UNESCO, 1979

BRAVO JUEGA, Isabel. La organización y gestión de museos. En: Boletín ANABAD, n I, 1995, pp. 177- 193
CARRIZO SAINERO, Gloria , IRURETA-GOYENA SÁNCHEZ, Pilar, LÓPEZ DE QUINTANA SÁENZ, Eugenio. Manual de fuentes de información. Madrid: Confederación de Gremios y Asociaciones de Libreros, 1994

CHECA, Fernando, GARCÍA, María de los Santos, MORÁN, José Miguel. Guía para el estudio de la Historia del Arte.

CICYT. Programa Nacional de Información para la Investigación científica y el Desarrollo tecnológico. Informe VICT00002-R07/89.04.20

COLL-VINENT, Robert. Información y poder. el futuro de las bases de datos documentales. Barcelona: Herder, 1988

COLL-VINENT, Roberto, BERNAL CRUZ, Francisco J. Curso de Documentación. Madrid: Dossat, 1990

CRUCES RODRÍGUEZ, Antonio. XIPNET: un proyecto integral de red informática distribuida para la difusión del Patrimonio cultural y artístico de la Comunidad valenciana. En: Catalogación del Patrimonio Histórico. Sevilla: Instituto Andaluz del Patrimonio Histórico, D.L. 1996, pp. 124-I28 (Cuadernos, VI)

DECRETO 107/89, de 16 de mayo, (BOJA 13/6/89) de creación del Instituto Andaluz del Patrimonio Histórico.

DELCLAUX, Ana Luis, CACHO, Carmen. Nuevas técnicas de documentación: Museo Arqueológico Nacional. En: Política Científica, n³4, 1992, pp. 38-41 
FUENTES de información en Ciencias Sociales y Humanidades. Madrid: CSIC, 1993

GONZÁLEZ DÍAZ DE GARAYO, C. , ROMÁN ROMÁN, A. Los problemas de información y documentación en las Humanidades. En: Revista Española de Documentación Científica, v. I, 1977, pp. $77-86$

INSTITUTO DE INFORMACIÓN Y DOCUMENTACIÓN EN CIENCIAS SOCIALES Y HUMANIDADES. Curso de fuentes documentales y recursos de información en ciencias humanas: Madrid, 22-24 de abril de 1991. Madrid: ISOC, 1991

LADRÓN DE GUEVARA SÁNCHEZ, Carmen. El Centro de Documentación del Patrimonio Histórico: objetivos, gestión y servicios. En: PH Boletín del Instituto Andaluz del Patrimonio Histórico, año IV, n I5, junio 1996, pp. I04- 109

LADRÓN DE GUEVARA SÁNCHEZ, Ma del Carmen. Hacia un Sistema de Información del Patrimonio Histórico. En: Patrimonio y ciudad. Sevilla: Instituto Andaluz del Patrimonio Histórico, 1994, pp. 76-8I (Cuadernos, V)

LADRÓN DE GUEVARA SÁNCHEZ, Ma del Carmen. Experiencias del Centro de Documentación: el Sistema de Información del Patrimonio Histórico (SIPHA). En: Catalogación del Patrimonio Histórico. Sevilla: Instituto Andaluz del Patrimonio Histórico, D.L. 1996, pp. 58-7I (Cuadernos, VI)

LEGISLACIÓN sobre el Patrimonio Histórico de Andalucía. Sevilla: Servicio de Publicaciones y BOJA de la Consejería de Presidencia. Junta de Andalucía, D.L. 1991 (Textos Legales , 2I)

LEY del Patrimonio Histórico Español y Reales Decretos de desarrollo parcial de la Ley. Madrid: Ministerio de Cultura, 1992

LÓPEZ YEPES, José. Fundamentos de información y documentación. Madrid: Eudema, 1989

LUCAS, Henry C. Sistemas de información: análisis, diseño, puesta a punto. Madrid: Paraninfo, 1987

MALDONADO MARTíNEZ, Angeles, FERNÁNDEZ, Elena. Las necesidades de información en los ámbitos de la arquitectura, el urbanismo y la ordenación del territorio: estudio de usuarios. En: Actas de las IV Jornadas Españolas de Documentación Automatizada. Gijón, 6-8 de octubre de 1994, pp. 659-666
MARTÍNEZ, L.J. Información y Documentación en el Plan Nacional de I+D: 1988-1993. En: Boletín de la ANABAD, n I, 1995, pp. 107-143

MUÑOZ COSME, Alfonso. Sistemas y metodología documental aplicada a los Bienes Culturales. En: Un proyecto para la CapiIla Real de Granada. Sevilla: Instituto Andaluz del Patrimonio Histórico, D.L. 1992, pp. 87-97 (Cuadernos , I)

OLMOS ROMERO, Ricardo. La investigación de la cultura material en los museos: notas para un debate. En: Boletín de la ANABAD, julio-septiembre 1988, XXXVIII, nº 3, págs. 9-I06

PLAN Andaluz de Investigación (1990-1993). Sevilla: Consejería de Educación y Ciencia, 1990

PLAN General de Bienes Culturales (1989-1995). Sevilla: Consejería de Cultura, D.L. 1993

PRAT CAROS, Joan...et al. Treinta años de literatura antropológica sobre España. En: Arxiu d'Etnografia de Catalunya, $1985-$ 1986, (45), pp. 9226

SABOR, Josefa. Manual de fuentes de información. Buenos Aires: Marymar, 1978

SANTIAGO, Elena. Fuentes para la historia del patrimonio artístico español en los archivos, bibliotecas y centros de documentación. En: Conferencia General de la IFLA, París, 16-19 de agosto de 1989

SCHOLOSSER, J.V. La literatura artística: manual de fuentes de la historia moderna del arte. Madrid, 1976

YARZA, J...et al. Fuentes y documentos para la historia del arte. Barcelona, 1982, 8 v. 